\title{
Kassenwechsel und Risikostrukturausgleich in der gesetzlichen Krankenversicherung - empirische Befunde der Kooperativen Gesundheitsforschung in der Region Augsburg (KORA)
}

\section{A. Werner ${ }^{1}$ \\ P. Reitmeir ${ }^{2}$ \\ J. John ${ }^{2}$}

Switching Sickness Funds and Risk Compensation Mechanisms in the Statutory

Health Insurance System in Germany - Empirical Results from the Cooperative

Health Research in the Region of Augsburg (KORA)

\section{Zusammenfassung}

Seit 1996 haben die in den gesetzlichen Krankenkassen versicherten Bürger der Bundesrepublik Deutschland das Recht zum Wechsel ihrer Krankenkasse. Ziel der Freigabe der Kassenwahl war es, Elemente der wettbewerblichen Steuerung im System der gesetzlichen Krankenversicherung zu stärken, um auf diese Weise eine effiziente und an den Präferenzen der Versicherten orientierte medizinische Versorgung zu gewährleisten. Um implizite Anreize zur Risikoselektion zu vermeiden, wurde gleichzeitig ein Risikostrukturausgleich zwischen den Krankenkassen eingeführt, der als morbiditätsbezogene Risikofaktoren Alter und Geschlecht der Versicherten sowie den Bezug von Erwerbs- oder Berufsunfähigkeitsrenten berücksichtigte. Auf der Grundlage des KORA-Surveys S4 (1999/2001) geht unsere Untersuchung am Beispiel des Kassenwechsels in der Region Augsburg während der Jahre 1996 bis 2001 der Frage nach, ob der Risikostrukturausgleich diese ihm zugedachte Funktion erfüllt hat. Die Untersuchungsergebnisse zeigen, dass Kassenwechsler auch nach Adjustierung auf Alter und Geschlecht seltener chronisch krank waren und seltener im Krankenhaus behandelt wurden als die übrigen Versicherten. Die unter dem geltenden Risikostrukturausgleich verbleibenden Beitragssatzunterschiede vermögen daher die Leistungs- und Wirtschaftlichkeitsunterschiede zwischen den Krankenkassen nicht korrekt zu reflektieren. Überdies gefährdet die Abwanderung vorzugsweise guter Risiken zu den beitragsgünstigen Krankenkassen das Prinzip der solidarischen Finanzierung. Der Risikostrukturausgleich bedarf daher einer die Risikostruktur der Versicherten genauer als bisher erfassenden Weiterentwicklung.

\section{Abstract}

Since 1996, all citizens of the Federal Republic of Germany who are insured in the statutory health insurance system are entitled to switch their sickness fund. The rationale of this regulation was to strengthen elements of competition in this system in order to stimulate the sickness funds to improve the efficiency of health care and to respond to consumers' preferences. Simultaneously, to avoid the implicit incentives for sickness funds to engage in risk selection, a risk compensation mechanism was introduced, including as morbidity-related risk adjusters age, sex and incapacity to work. Based on the KORA survey S4 (1999/2001) we take the case of switching behaviour in the region of Augsburg, and analyse whether this risk adjustment scheme was working effectively. The results show that persons changing their sickness fund were characterised by a comparatively smaller burden of chronic diseases and by a less frequent utilization of inpatient health care. Under these conditions, differences in the contribution rates do not accurately reflect differences in the performance and efficiency of sickness funds. Moreover, the migration of good risk to sickness funds with favourable contribution rates threatens the principle of financial solidarity. Therefore, the system of risk equalisation has to be developed towards measuring the risk volume borne by the sickness funds more precisely than hitherto.

Key words

Social health insurance $\cdot$ competition $\cdot$ health plan choice $\cdot$ risk compensation $\cdot$ adverse selection

${ }^{1}$ Kreiskrankenhaus Weilheim, Weilheim

${ }^{2}$ GSF - Forschungszentrum für Umwelt und Gesundheit, Institut für Gesundheitsökonomie und Management im Gesundheitswesen (IGM), Neuherberg

Korrespondenzadresse

Dr. Jürgen John · GSF - Forschungszentrum für Umwelt und Gesundheit, Institut für Gesundheitsökonomie und Management im Gesundheitswesen·Postfach 1129·85758 Neuherberg·E-mail: john@gsf.de

Bibliografie

Gesundheitswesen 2005; 67 Sonderheft 1: S158 -S166 @ Georg Thieme Verlag KG Stuttgart • New York DOI 10.1055/s-2005-858261

ISSN 0949-7013 


\section{Schlüsselwörter}

Soziale Krankenversicherung · Wettbewerb · Kassenwahl · Risikoausgleich · adverse Selektion

\section{Hintergrund und Forschungsstand}

Die Steuerung des Systems der gesetzlichen Krankenversicherung (GKV) erfolgte bis zu Beginn der 90er-Jahre überwiegend durch administrative und kollektivvertragliche Instrumente wie Planung, Budgetierung oder Honorarvereinbarungen. Wachsende Zweifel an der Effizienz und Innovationsfähigkeit des Systems veranlassten den Gesetzgeber dazu, mit dem Gesundheitsstrukturgesetz von 1992 den Prozess einer Transformation des dirigistischen Ordnungsrahmens der GKV in eine „solidarische Wettbewerbsordnung“ zu eröffnen. Ziel dieser Ordnung ist es, das als unverzichtbar geltende Prinzip solidarisch finanzierter und gleichmäßiger Versorgung der Versicherten im Krankheitsfall mit dem Prinzip markt- und wettbewerblicher Steuerung zu verknüpfen. Zwei Elemente der Strukturreform sind in unserem $\mathrm{Zu}$ sammenhang von besonderem Interesse:

- die Einführung des Rechts der freien Kassenwahl für den überwiegenden Teil der GKV-Versicherten, das das alte Zuweisungsrecht ablöste, nach dem die meisten Mitglieder der GKV in Abhängigkeit von Charakteristika ihres Arbeitsplatzes einer bestimmten Krankenkasse zugewiesen wurden, sowie

- die Einführung eines dauerhaften kassen- bzw. kassenartenübergreifenden Risikostrukturausgleichs (RSA), der auf der Einnahmenseite die beitragspflichtigen Einnahmen der Mitglieder und auf der Ausgabenseite die Familienlast (Anzahl der beitragsfrei versicherten Familienangehörigen), Alter und Geschlecht, Invalidität in Form des Bezugs einer Erwerbsoder Berufsunfähigkeitsrente sowie den Umfang des Krankengeldanspruchs als Risikofaktoren einbezog (zu Einzelheiten und Weiterentwicklungen des RSA siehe [1]).

Die Freigabe der Kassenwahl zielte auf die Etablierung des Wettbewerbs zwischen den Kassen zur Erreichung einer bedarfsgerechten, qualitäts- und innovationsorientierten sowie effizienten Gesundheitsversorgung der Versicherten. Der RSA sollte zum einen dazu dienen, den Kassenwettbewerb auf diese zentralen gesundheitspolitischen Ziele auszurichten und den Wettbewerb um „gute Risiken“ unattraktiv, da finanziell nicht lohnend, zu machen, zum anderen sollten dadurch alle Beitragszahler im gewünschte Maße, d.h., entsprechend ihrer wirtschaftlichen Leistungsfähigkeit, zur Finanzierung der Gesundheitsversorgung herangezogen werden. Schließlich sollte der RSA auch annähernd gleiche Startchancen für alle Kassen im Wettbewerb herstellen, da die Risikostrukturen und demzufolge auch die Beitragssätze zwischen den Einzelkassen bzw. den Kassenarten als Folge des alten Mitgliedschaftsrechts stark divergierten.

Vorliegende empirische Analysen zur Funktion des RSA zeigen, dass dieser die rein versichertenstrukturbedingten Unterschiede in den Beitragssätzen deutlich verkleinert und insoweit auch Selektionsanreize vermindert hat. Statische Simulationsrechnungen zeigen, dass für Krankenkassen mit hoher Finanzkraft und niedrigem Beitragsbedarf der kostendeckende Beitragssatz 1999 im Durchschnitt bei 7,5\% - in zwei extremen Einzelfällen sogar unter 3\% - gelegen hätte, während für Krankenkassen mit niedriger Finanzkraft und hohem Beitragsbedarf der ausgabendeckende Beitragssatz im Mittel über 20\% betragen hätte. Der RSA hat die Unterschiede zwischen den mittleren Beitragssätzen in diesen beiden Extremgruppen auf rund 2 Prozentpunkte reduziert [1]. Gleichzeitig ist aber auch die Vermutung geäußert worden, dass die Beitragssatzunterschiede auch in den letzten Jahren immer noch wesentlich durch den mangelnden Ausgleich des Morbiditätsrisikos verursacht werden. Risikoselektion - sei es durch aktive Maßnahmen seitens der Kassen, sei es durch Selbstselektion der Versicherten - verbessere daher weiterhin die Wettbewerbsposition einer Kasse. Trifft dieser Verdacht zu, so gefährdet dies die solidarische Wettbewerbsordnung in doppelter Weise: Erstens, solange Beitragssatzunterschiede nicht primär Indikatoren unterschiedlicher Leistungsfähigkeit und Effizienz sind, sondern vor allem bessere oder schlechtere Risikostrukturen abbilden, verzerren sie den Wettbewerb und lenken ihn verstärkt auf den volkswirtschaftlich unsinnigen Kampf um „gute Risiken“. Zweitens entziehen sich Versichertengemeinschaften mit günstiger Risikostruktur in dem Ausmaß, in dem diese nicht im RSA berücksichtigt wird, dem politisch gewünschten GKV-weiten Solidarausgleich. Eine morbiditätsortientierte Weiterentwicklung des RSA ist daher nach Einschätzung vieler Beobachter (z.B. [2, 3]) dringend geboten, wobei überwiegend zugunsten von diagnosegestützten Kompensationsmechanismen votiert wird (zu einem Überblick siehe $[4,5]$ ); vereinzelt sind aber durchaus auch andere Adjustierungsverfahren zur Diskussion gestellt worden (z.B. [6, 7]).Es ist daher nicht nur wissenschaftlich, sondern auch politisch von eminentem Interesse, die Frage zu klären, ob die durch die Freigabe der Kassenwahl ausgelösten Wechselbewegungen zwischen den Krankenkassen durch Risikoselektion und zunehmende Risikosegmentierung zwischen den Kassen charakterisiert sind. Der KORA-Survey 1999/2001 (S4), ein Gesundheitssurvey mit breitem Erhebungsprogramm morbiditäts- und gesundheitsbezogener Daten, bot sich hierfür als geeigneter empirischer Zugang an.

Erste empirische Analysen der Auswirkungen der erweiterten Kassenwahlrechte stützten sich auf Aggregatdaten aus den Mitgliederstatistiken der GKV und der RSA-Finanzstatistik [8-10]. Die Untersuchungen weisen u.a. aus, dass Mitgliederbestandsbewegungen bzw. die Entwicklung der Mitgliedermarktanteile der einzelnen Kassenarten in den drei ersten Jahren nach Inkraft-Treten der erweiterten Kassenwahlrecht eng mit den Beitragssätzen zusammenhingen, dass Änderungen der Mitgliederzahlen in der Krankenversicherung der Rentner (KVdR) auch nach Einführung kassenspezifischer Beitragssätze für Rentner weitaus geringer als in der Allgemeinen Krankenversicherung (AKV) waren, und dass das RSA-Transfervolumen von Jahr zu Jahr anwuchs. Wirtschaftswissenschaftlich orientierte Aggregatdatenanalysen haben vor allem den Einfluss der Beitragssätze auf die Mitgliederentwicklung der Kassen bzw. die Kassenwahlentscheidungen thematisiert. In verschiedenen Studien ist für die zweite Hälfte der 90er-Jahre mit Hilfe von Korrelationsoder Regressionsanalysen sowohl auf der Ebene von Kassenarten als auch auf der Ebene einzelner Krankenkassen der Zusammenhang zwischen Mitgliederbestandsbewegungen und Beitragssätzen statistisch belegt worden [11-14]. Schut et al. [15] haben schließlich für den Zeitraum von 1996 bis 2001 wiederum unter 
Nutzung von Aggregatdaten auf der Ebene von Kassenarten Preiselastizitäten der Kassenmarktanteile geschätzt. Dabei ergaben sich für AKV-Mitglieder im gesamten Zeitraum statistisch signifikante und im Zeitverlauf zunehmende Preiselastizitäten. Insgesamt stützen die makrostatistischen Befunde die teilweise schon im Vorfeld des Inkrafttretens des neuen Kassenwahlrechts geäußerten Vermutungen, dass die Beitragssätze der Krankenkassen wesentliche Determinanten individueller Kassenwahlentscheidungen sind, dass die Neigung zum Kassenwechsel mit zunehmendem Alter abnimmt und dass die Kassenwechselbewegungen von einer Risikoentmischung zwischen den Kassenarten in den Charakteristika der Versicherten begleitet werden, die im geltenden RSA bereits berücksichtigt werden. Für eine Beurteilung der im Kontext der Debatte über die Weiterentwicklung des RSA entscheidenden Frage, ob der RSA seiner Aufgabe gerecht wird, Selektionseffekte des Kassenwechsels zu neutralisieren und den Wettbewerb auf seine eigentliche Funktion als Mittel zur Steigerung der Versorgungseffizienz und -qualität zu orientieren, bieten die vorhandenen makrostatistischen Daten und die darauf aufbauenden Untersuchungen hingegen keine geeignete Grundlage.

Den bisher publizierten mikroanalytischen Untersuchungen von Kassenwahlentscheidungen nach Erweiterung des Kassenwahlrechts mit dem Ziel, die Motive für solche Entscheidungen aufzudecken und die sich abzeichnenden Wechseltendenzen in ihren Auswirkungen auf die Risikostrukturen der einzelnen Kassenarten abzuschätzen, ist gemeinsam, dass sie alle Daten des Sozioökonomischen Panels (SOEP) aus den Jahren 1997 bis 2000 nutzten [16 - 19]. Ein einheitliches Bild ergab sich in diesen Untersuchungen für den Einfluss der Beitragssätze; je höher diese waren, desto höher war die Wahrscheinlichkeit eines Kassenwechels der Versicherten. Instabile und teilweise widersprüchliche Resultate erbrachten die Studien hingegen in Hinblick auf die untersuchten Zusammenhänge zwischen Gesundheitszustand bzw. Inanspruchnahme der medizinischen Versorgung und Kassenwechsel. So war ein im Eigenurteil guter Gesundheitszustand nach Altersadjustierung teils mit höherer [18, 19], teils aber auch mit niedrigerer Wechselwahrscheinlichkeit [17] als bei vergleichsweise schlechterer Gesundheit assoziiert. Die altersadjustierte Inanspruchnahme der medizinischen Versorgung deutete nur im Falle der stationären Behandlung, nicht aber im Falle der ambulanten Behandlung darauf hin, dass Kassenwechsler „bessere Risiken“ waren; überdies waren auch die Ergebnisse für die Krankenhausbehandlung nicht für alle untersuchten Jahre statistisch auffällig $[17,18]$.

In einigen Studien wurde versucht, die mit dem Kassenwechsel möglicherweise einhergehende Risikoentmischung in ihren finanziellen Dimensionen zu quantifizieren, indem auf Basis von GKV-Routinedaten der „relative Beitragsbedarf“ der Wechsler berechnet wurde; unter dem relativen Beitragsbedarf ist die Relation der tatsächlichen Leistungsausgaben für diese Personengruppe zu den mittleren („standardisierten“) Leistungsausgaben zu verstehen, wie sie sich im RSA unter Berücksichtigung von Alter, Geschlecht und der weiteren in diesem Ausgleichsverfahren herangezogenen Personenmerkmale für die Versicherten insgesamt ergeben [20, 21]. Lauterbach und Wille [20] haben solche Berechnungen auf Basis von Daten der Jahre 1998 und 1999 für insgesamt acht teilweise sehr mitgliederstarke Krankenkassen durchgeführt. Die einbezogenen Leistungsbereiche umfassen für beide Jahre die Arzneimittelversorgung, das Krankengeld und die Krankenhausbehandlung, für 1999 darüber hinaus einen nicht näher spezifizierten Bereich „sonstiger“ Leistungen, mangels versichertenbeziehbarer Daten aber nicht die vertragsärztliche und die vertragszahnärztliche Versorgung. Die Berechnungen ergaben, dass Wechsler im Jahr vor ihrem Wechsel in allen Leistungsbereichen und Altersgruppen Leistungsausgaben aufwiesen, die unter dem Beitragsbedarf ihrer jeweiligen RSA-Gruppe lagen. Die Wechsler wiesen für die erfassten Leistungen insgesamt positive Deckungsbeträge von durchschnittlich etwa 600 DM auf, was einem relativen Beitragsbedarf von etwa $60 \%$ entsprach. Eine Differenzierung nach Altersgruppen ergab keine systematische Altersabhängigkeit des relativen Beitragsbedarfs der Wechsler und dementsprechend ein deutliches Wachstum der positiven Deckungsbeiträge mit zunehmendem Alter. Winkelhake et al. [21] kommen zu ähnlichen Ergebnissen. Die Aussagekraft beider Studien findet eine wesentliche Grenze u. a. darin, dass offen bleibt, in welchem Umfang und wie lange Wechsler auch nach ihrem Wechsel weniger Leistungsausgaben verursachen, als die aufnehmenden Krankenkassen für die neuen Versicherten an Beitragsbedarf erhalten.

Einen ähnlichen Versuch zur Bestimmung der Risikoprofile der Kassenwechsler haben Andersen et al. [22] auf der Basis einer Verknüpfung der RSA-Daten zu den standardisierten Leistungsausgaben in den Hauptleistungsbereichen Ärzte und Krankenhaus mit Daten aus dem SOEP für die Jahre 1997 bis 2001 unternommen. Die fünf Querschnittsanalysen ergaben deutliche Unterschiede zwischen den alten und den neuen Bundesländern: Während Wechsler in den neuen Bundesländern mit einem durchschnittlichen Beitragsbedarf von ca. $80 \%$ als gute Risiken identifiziert wurden, traf dies auf die Wechsler in den alten Bundesländern - das Jahr 1999 ausgenommen - mit einem relativen Beitragsbedarf von durchschnittlich etwas mehr als 100\% nicht zu. Eine Tendenz zur Risikoentmischung als Folge von Mitgliederbewegungen zwischen den Kassen zeigte sich erst bei Differenzierung der Wechsler nach den aufnehmenden Kassenarten, die ergab, dass gute Risiken vorwiegend zu den Krankenkassen mit eher niedrigen Beitragssätzen (BKK, IKK) und schlechte Risiken überwiegend zu Krankenkassen mit eher hohen Beitragssätzen (AOK, EK) migrierten.

Zusammenfassend lässt sich festhalten, dass sich auf Grundlage der bisher publizierten Studien über Unterschiede in den Risikoprofilen zwischen Wechslern und Nichtwechslern keine eindeutige Evidenzlage zugunsten oder zuungunsten der Hypothese ableiten lässt, dass Kassenwechsel mit adverser Selektion einhergeht.

\section{Methodik}

Die Studienpopulation des KORA-Surveys S4 ist eine repräsentative Stichprobe der Bevölkerung deutscher Staatsangehörigkeit im Alter von 25 bis 74 Jahren mit Wohnsitz in der Studienregion Augsburg, die die kreisfreie Stadt Augsburg sowie die beiden angrenzenden Landkreis Augsburg und Aichach-Friedberg umfasst. Die Stichprobe von brutto insgesamt 6640 Personen (ca. 1,5\% der Grundgesamtheit) wurde in einem Clustersamplingverfahren 
aus den Einwohnern der Stadt Augsburg und von 16 Umlandgemeinden gezogen, wobei eine Stratifizierung in 10 gleich große Strata von je 664 Personen nach Geschlecht und Alter (jeweils fünf 10-Jahres-Altersgruppen) vorgenommen wurde. Aus der Stichprobe fielen insgesamt 260 Personen als neutrale Ausfälle wegen Tod, Umzug oder unzureichender Deutschkenntnisse heraus, sodass die bereinigte Bruttostichprobe 6380 Personen umfasste. Von diesen nahmen insgesamt 4261 Personen am Survey teil, was einer Responserate von 66,8\% entspricht.

Der Survey, der von Oktober 1999 bis April 2001 durchgeführt wurde, umfasste ein umfangreiches Programm von Untersuchungen und Messungen sowie ein ausführliches Interview. Mit Ausnahme der Beitragssätze, die im Jahr 2002 in einer schriftlichen Erhebung direkt bei den Krankenkassen erhoben wurden, da sie der überwiegenden Mehrzahl der Studienteilnehmer nicht präsent waren, entstammen alle in unserer Untersuchung verwendeten Daten diesem Survey. Nicht alle Probanden hatten die Zeit oder die Möglichkeit, das gesamte Erhebungsprogramm zu absolvieren, sodass zur Durchführung der Befragung im Rahmen des Teilvorhabens „Kassenwahl“ 4131 Personen zur Verfügung standen. Zielpopulation des Teilvorhabens waren alle Studienteilnehmer, die gesetzlich oder privat krankenversichert waren. 45 Personen erfüllten diese Voraussetzung nicht, sodass die Zielpopulation - im Folgenden ,Studienpopulation A' genannt - 4086 Personen umfasste. Für die Analyse der Auswirkungen der zum 1.1.1996 eingeführten freien Kassenwahl wurde eine zweite Untersuchungspopulation - im Folgenden ,Studienpopulation B' genannt - definiert, die alle Studienteilnehmer umfasste, die zum Zeitpunkt des Interviews in einer gesetzlichen Krankenkasse versichert waren und die zudem (a) in dieser Kasse auch schon vor Jahresbeginn 1996 versichert gewesen waren oder (b) nach dem 31.12.1995 aus einer anderen gesetzlichen Krankenkasse in ihre derzeitige Kasse gewechselt waren; die letztere Gruppe umfasst die „Kassenwechsler“ in Sinne unserer Studie. Ausgeschlossen aus Studienpopulation B waren somit privat versicherte Probanden sowie gesetzlich versicherte Studienteilnehmer, die nach dem 31.12.1995 aus einer privaten Krankenversicherung in ihre derzeitige gesetzliche Kasse gewechselt waren. Studienpopulation B umfasste 3341 Personen.

\section{Ergebnisse}

\section{Umfang und Struktur des Kassenwechsels}

Nimmt man die beiden großen Systeme der kollektiven finanziellen Absicherung des Krankheitsrisikos, GKV und private Krankenversicherung (PKV), gemeinsam in den Blick, so hat die Studie ergeben, dass im Beobachtungszeitraum zwischen dem 1.1.1996 und dem Zeitpunkt ihrer Befragung insgesamt 586 Studienteilnehmer ihre Krankenkasse oder Krankenversicherung gewechselt haben (Tab.1); dies sind 14,3\% aller 4086 Probanden der Studienpopulation A.

453 oder gut drei Viertel aller Wechselfälle sind Binnenwanderungen innerhalb der Systeme. Die Binnenwanderungsfrequenz ist in der GKV weitaus höher als in der PKV. Dies mag zum Teil an einem mitgliedschaftsrechtlich bedingten Aufstau von Wechselwünschen in der GKV liegen, der sich erst ab 1996
Tab. 1 Wechsler zwischen den Systemen der gesetzlichen (GKV) und der privaten (PKV) Krankenversicherung sowie innerhalb der beiden Systeme im KORA-Survey S4 (1999/2001)

\begin{tabular}{llll}
\hline \multicolumn{2}{l}{ Wechsel zu ... } & & \\
Wechsel von ... & GKV & PKV & GKV+PKV \\
\hline GKV & 424 & 73 & 497 \\
& $(72,4 \%)^{1} /[12,5 \%]^{2}$ & $(12,5 \%) /[10,7 \%]$ & $(84,8 \%)$ \\
\hline PKV & 60 & 29 & 89 \\
& $(10,2 \%) /[1,7 \%]$ & $(4,9 \%) /([4,2 \%]$ & $(15,2 \%)$ \\
GKV + PKV & 484 & 102 & 586 \\
& $(82,6 \%) /[14,2 \%]$ & $(17,4 \%) /[14,9 \%]$ & $(100,0 \%)$ \\
\hline
\end{tabular}

${ }^{1}$ Zahlen in runden Klammern: Anteilswerte der einzelnen Wechslergruppen an den Wechslern insgesamt

${ }^{2}$ Zahlen in eckigen Klammern: Anteilswerte der einzelnen Wechslergruppen an den Versichertenbeständen von GKV und PKV in Studienpopulation A zur Zeit der Befragung

abbauen konnte, und zu dem es in der PKV keine Parallele gibt. Außerdem ist zu bedenken, dass Altersrückstellungen beim Wechsel einer privaten Krankenversicherung nicht mitgenommen werden können, was dazu führt, dass ein Versicherungswechsel mit zunehmendem Alter schnell finanziell unattraktiv wird und dass sich der Wettbewerb der Krankenversicherer daher auf die Neukunden konzentriert. 133 oder ein knappes Viertel der Wechselbewegungen entfallen auf Wanderungen zwischen GKV und PKV; der Wanderungssaldo zugunsten der PKV entspricht in seiner relativen Größenordnung recht gut den für die Bundesrepublik insgesamt beobachteten Daten der letzten Jahre. Die Wechselbewegungen zwischen den Systemen liegen in einer Größenordnung, die es nicht erlauben, Einflüsse der Außenwanderungen auf die Risikostrukturen der Krankenkassen von vornherein auszuschließen. Die Liberalisierung des Mitgliedschaftsrechts der GKV hat jedoch an den Spielräumen für ein „opting in“ in die GKV und ein „opting out“ aus der GKV nichts geändert, sodass diese Wechselbewegungen im Kontext des Themas „freie Kassenwahl und Risikostrukturausgleich“ nicht weiter zu thematisieren sind.

Einen Überblick über Umfang und Richtung des Kassenwechsels innerhalb der GKV gibt auf der Ebene der Kassenarten Tab. 2. Von den 3341 Studienteilnehmern, die schon vor Inkrafttreten des neuen Mitgliedschaftsrechts am 1.1.1996 der GKV angehörten, wechselten bis zum Zeitpunkt ihrer Befragung im Rahmen des KORA-Surveys S4 424 (= 12,7\%) ihre Krankenkasse.

Für Orts-, Ersatz- und insbesondere Innungskrankenkassen sind per Saldo Versichertenverluste zu verzeichnen; dem steht eine Zunahme der bei einer Betriebskrankenkasse versicherten Probanden im Vergleich zum initialen Versichertenbestand in der Studienpopulation B um ein Drittel gegenüber. Zur Kategorie der sonstigen Krankenkassen zählen die Landwirtschaftlichen Krankenkassen, die Seekasse und die Bundesknappschaft. Für diese berufsständisch orientierten Krankenkassen gilt das Recht der freien Kassenwahl nicht; die Zahlen zu den in diesen Fällen in der Regel rein mitgliedschaftsrechtlich begründeten $\mathrm{Zu}$ - und $\mathrm{Ab}$ wanderungen deuten darauf hin, dass ein kassenartenüberschreitender Kassenwechsel auch im Rahmen des alten Zuweisungsrechts durchaus kein ganz seltener Vorgang ist bzw. war. 


\begin{tabular}{lrlllllll}
\hline $\begin{array}{l}\text { initialer Versichertenbestand (IVB) } \\
\text { nach Kassenart } \\
\text { Kassenart }\end{array}$ & $\boldsymbol{N}$ & $\boldsymbol{n}$ & $\begin{array}{l}\text { Abwanderung } \\
\text { in \% des } \\
\text { IVB }\end{array}$ & $\boldsymbol{n}$ & $\begin{array}{l}\text { Zuwanderung } \\
\text { in des } \\
\text { IVB }\end{array}$ & \multicolumn{2}{c}{$\begin{array}{l}\text { Bestandsänderung } \\
\text { in \% des } \\
\text { IVB }\end{array}$} \\
\hline Ortskrankenkassen & 1029 & -116 & $-11,3$ & 47 & 4,6 & -69 & $-6,7$ \\
\hline Ersatzkrankenkassen & 1321 & -205 & $-15,5$ & 81 & 6,1 & -124 & $-9,4$ \\
\hline Betriebskrankenkassen & 668 & -37 & $-5,5$ & 260 & 38,9 & +223 & $+33,4$ \\
\hline Innungskrankenkassen & 259 & -63 & $-24,3$ & 22 & 8,5 & -41 & $-15,8$ \\
\hline sonstige Krankenkassen & 64 & -3 & $-4,7$ & 14 & 21,9 & +11 & $+17,2$ \\
\hline insgesamt & 3341 & -424 & & 424 & & 0 & \\
\hline
\end{tabular}

Tab. 2 Kassenwanderung der Teilnehmer im KORA-Survey S4 (1999/2001) innerhalb der gesetzlichen Krankenversicherung nach Kassenarten

Eine demographische Betrachtung der Struktur der Kassenwanderung ergibt für geschlechtsspezifische Unterschiede in der Häufigkeit des Kassenwechsels keine Anhaltspunkte; dies gilt sowohl für einen Vergleich von Männern und Frauen insgesamt als auch für eine nach Altersgruppen stratifizierte Betrachtung. Eine nach dem Alter der Probanden differenzierte Betrachtung zeigt hingegen massive Unterschiede in der Wechselhäufigkeit zwischen den einzelnen Altersgruppen auf (Abb.1).

Der Anteil der Wechsler unter den Probanden nahm mit zunehmendem Alter stetig und stark ab. Während in der Altersgruppe der 25- bis 34-jährigen Versicherten nahezu jeder Dritte seine Krankenkasse gewechselt hatte, traf dies in der Altersgruppe der 65- bis 74-jährigen Versicherten nur auf etwa jeden hundertsten Probanden zu. Es ist schwer zu beurteilen, wie weit diese großen Unterschiede Ausdruck von Alters- oder Kohorteneffekten sind. Die Existenz eines Alterseffekts ist schon aufgrund des empirisch gut bestätigten Sachverhalts einer mit wachsendem Alter zunehmenden Risikoaversion als sicher anzunehmen. Andererseits dürfte ein Kohorteneffekt insofern eine Rolle spielen, als für Rentner ein Kassenwechsel erst ab Mitte 1999 finanziell attraktiv werden konnte, da bis dahin für die Krankenversicherung der Rentner in allen Krankenkassen der gleiche Beitragssatz erhoben wurde. Zudem lehrt die Beobachtung anderer Märkte, wie z.B. des Markts für Telefondienstleistungen, dass sich jüngere Menschen an technische und institutionelle Änderungen schneller als die Älteren anpassen. Auf längere Sicht wird daher mit einer Abflachung des Altersgradienten zu rechnen sein.

\section{Kassenwechsel und Risikoselektion}

Die Beantwortung der Frage, ob Kassenwechsel zu einer Risikoentmischung zwischen den Krankenkassen führt, erfordert die

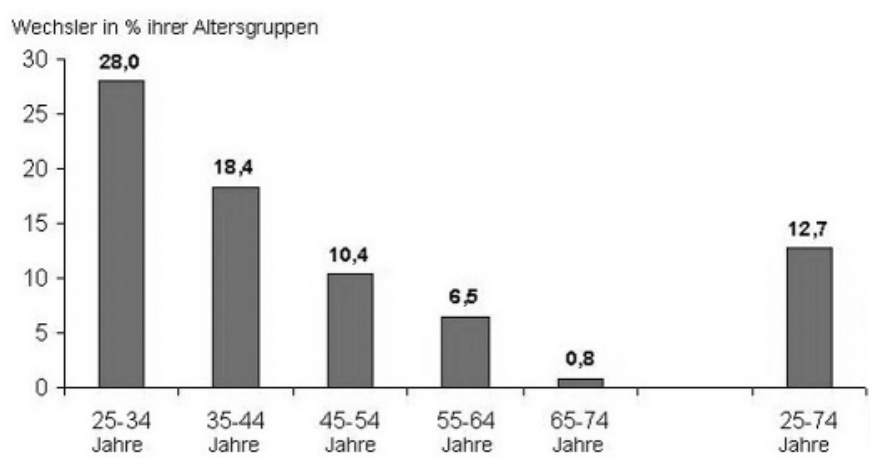

Abb. 1 Kassenwechsler in Prozent aller GKV-Versicherten der gleichen Altersgruppe im KORA-Survey $\$ 4$.
Untersuchung der Frage, ob sich Kassenwechsler in ihrer Risikostruktur von den Nichtwechslern unterscheiden. Tab. 3 gibt eine Übersicht über die Ausprägungen einer Reihe von Merkmalen in den beiden miteinander zu vergleichenden Gruppen, die als Risikoindikatoren gelten können. Wechsler und Nichtwechsler unterscheiden sich in nahezu allen dieser Merkmale voneinander: Wechsler sind deutlich jünger als Nichtwechsler, ihr Haushaltseinkommen ist höher, sie haben häufiger einen höheren Ausbildungsabschluss, sie haben einen niedrigeren Bodymass-Index und sind körperlich aktiver, sie nehmen weniger medizinische Versorgungsleistungen in Anspruch und sind seltener krank. Neben einem nahezu gleichen Frauenanteil sind sich beide Gruppen auch im mittleren Beitragssatz zum Zeitpunkt des Inkrafttretens der erweiterten Kassenwahlrechte ähnlich, was angesichts des in vielen Studien belegten hohen Einflusses des Beitragssatzes auf die Wechselwahrscheinlichkeit zunächst überraschend erscheinen mag; hierauf wird später zurückzukommen sein. Der prägende Einfluss der Beitragssätze bzw. Beitragssatzunterschiede auf die Richtung der Wanderungsströme wird allerdings schon anhand der Beitragssätze am Ende der Beobachtungsperiode sichtbar: Am 1.1.2001 galt für die Kassenwechsler im Durchschnitt ein Beitragssatz von 12,9\%, für die Nichtwechsler ein Beitragssatz von 13,7\%. Die Wechsler haben also in diesem 5-Jahreszeitraum im Schnitt einen Beitragsrückgang von -0,1 Prozentpunkten realisiert, während die Nichtwechsler einen Anstieg ihrer Beitragssätze von durchschnittlich 0,8 Prozentpunkten hinnehmen mussten.

Die in Tab. 3 zusammengestellten Befunde lassen keinen Zweifel daran, dass die Kassenwechsler in unserem Untersuchungszeitraum im Vergleich zu den Nichtwechslern die besseren Risiken waren. Unklar ist freilich, ob der geltende RSA Lücken in der Risikokompensation lässt, denn mit dem Alter ist ein Risikofaktor im RSA berücksichtigt, der mit den anderen aufgelisteten Risikofaktoren eng korreliert; dies gilt wegen seines positiven Langzeittrends selbst für den höchsten Ausbildungsabschluss.

Zur Untersuchung der Frage, ob die in den RSA einbezogenen Faktoren die Risikounterschiede zwischen den beiden Gruppen ausgleichen, wurde eine logistische Regressionsanalyse durchgeführt. Hierfür wurden alle Variablen des Datensatzes des KORA-Surveys $\mathrm{S} 4$ herangezogen, die als Indikatoren des Morbiditätsrisikos betrachtet werden können oder die als sonstige - vermutete oder empirisch schon bestätigte - Determinanten der Wahrscheinlichkeit eines Kassenwechsels anzusehen sind. Der analysierte Datensatz umfasst im Einzelnen Merkmale der Soziodemografie einschließlich der in Hinblick auf die beitragsfreie Familienversicherung relevanten Familienzusammensetzung, 
Tab. 3 Wechsler- und Nichtwechslerpopulation im KORA-Survey S4 (1999/2001) nach ausgewählten Merkmalen

\begin{tabular}{|c|c|c|}
\hline Merkmal & $\begin{array}{l}\text { Wechsler } \\
(n=424)\end{array}$ & $\begin{array}{l}\text { Nichtwechsler } \\
(n=2917)\end{array}$ \\
\hline Alter in Jahren & 39,9 & 51,7 \\
\hline Altersgruppe 25 - 34 Jahre in \% & 43,8 & 16,4 \\
\hline Altersgruppe $35-44$ Jahre in \% & 28,1 & 18,2 \\
\hline Altersgruppe $45-54$ Jahre in \% & 16,0 & 20,2 \\
\hline Altersgruppe $55-64$ Jahre in $\%$ & 10,9 & 22,8 \\
\hline Altersgruppe $65-74$ Jahre in $\%$ & 1,2 & 22,4 \\
\hline Anteil Frauen in \% & 54,7 & 53,7 \\
\hline $\begin{array}{l}\text { Anzahl beitragsfrei mitversicherter Familien- } \\
\text { angehöriger }\end{array}$ & 0,55 & 0,47 \\
\hline Beitragssatz am 1.1.1996 in \% & 13,0 & 12,9 \\
\hline Beitragssatz am 1.1. 2001 in \% & 12,9 & 13,7 \\
\hline größter Beitragssprung seit dem 1.1.1996 & 0,76 & 0,66 \\
\hline Haushaltsnettoeinkommen in DM & 5858 & 5466 \\
\hline $\begin{array}{l}\text { Anteil Personen mit Abitur/Hochschulabschluss } \\
\text { in } \%\end{array}$ & 24,1 & 15,3 \\
\hline Anzahl Ausbildungsjahre & 11,7 & 11,1 \\
\hline Sozialschicht-Index & 3,0 & 2,7 \\
\hline Bodymass-Index & 2,75 & 3,04 \\
\hline Anteil der körperlich nicht aktiven Personen in \% & 27,6 & 37,0 \\
\hline Anteil der Nieraucher & 41,5 & 43,1 \\
\hline Anzahl Arztbesuche in den letzten 4 Wochen & 0,66 & 0,81 \\
\hline $\begin{array}{l}\text { Anteil Personen mit Krankenhausaufenthalt in } \\
\text { den letzten } 12 \text { Monaten }\end{array}$ & 9,43 & 14,23 \\
\hline $\begin{array}{l}\text { Anzahl Krankenhausaufenthalte in den letzten } \\
12 \text { Monaten }\end{array}$ & 0,11 & 0,18 \\
\hline Anteil Probanden mit chronischer Bronchitis in \% & 3,07 & 8,56 \\
\hline Anteil Probanden mit Herzinfarkt in \% & 0,94 & 3,69 \\
\hline Anteil Probanden mit Schlaganfall in \% & 0,24 & 2,19 \\
\hline Anteil Probanden mit Diabetes in \% & 1,20 & 4,88 \\
\hline Anteil Probanden mit Varicosis in \% & 6,64 & 15,35 \\
\hline $\begin{array}{l}\text { Anteil Probanden mit arterieller Verschluss- } \\
\text { krankheit in \% }\end{array}$ & 2,12 & 5,00 \\
\hline Anteil Probanden mit Herzinsuffizienz in \% & 0,71 & 3,74 \\
\hline Anteil Patienten mit Arthritis & 6,43 & 9,54 \\
\hline $\begin{array}{l}\text { Anteil Probanden mit mindestens einer chro- } \\
\text { nischen Krankheit in \% }\end{array}$ & 58,7 & 75,8 \\
\hline
\end{tabular}

Kassenmerkmale einschließlich der Höhe und der Veränderung des Beitragssatzes, Merkmale der Inanspruchnahme des Gesundheitswesens, die traditionellen epidemiologischen Verhaltensrisikofaktoren, die teils durch Befragung (Rauchen, Alkoholkonsum, gesunde Ernährung, körperliche Aktivität), teils durch anthropometrische Messungen (Bodymass-Index) gewonnen wurden sowie Merkmale der Morbidität, die in Form einer Eigenanamnese sowie einer Blutdruckmessung erhoben wurden.

Die statistische Auswertungsstrategie bestand in einer multivariablen logistischen Regressionsanalyse mit der binären Zielgröße „Kassenwechsel (ja/nein)“ als abhängige Variable, in der in einem ersten Schritt alle einbezogenen Faktoren im Rahmen des Tests von fünf Untermodellen (Soziodemografie, Inanspruchnahme, Erkrankungsrisiken, Kassenmerkmale, Morbidität) einem Variablenscreening unterzogen wurden. Alter und Geschlecht der Probanden wurden dabei in allen Untermodellen eingeschlossen, um auf diesbezügliche Einflüsse zu kontrollieren. In einem zweiten Schritt wurden alle Variablen, die sich in den Untermodellen als statistisch auffällige Unterscheidungsmerkmale zwischen Wechslern und Nichtwechslern erwiesen hatten, in ein Endmodell überführt und gemeinsam getestet. In den Untermodellen wurde ebenso wie im Endmodell die Variablenselektion sowohl mittels einer Forward- als auch mittels einer Backward-Stepping-Prozedur unter Zugrundelegung eines Schrankenwerts von $\mathrm{p}=0,05$ durchgeführt. Es kann vorweggenommen werden, dass beide Prozeduren in allen Modellen jeweils zur gleichen Variablenauswahl führten, was verdeutlicht, dass keine bedeutsamen Multikollinearitätsprobleme auftraten.

Das Untermodell Soziodemografie umfasste das Haushaltseinkommen, den höchsten Schulabschluss sowie die Anzahl der Ausbildungsjahre als übliche Indikatoren der Sozialschichtzugehörigkeit. Für keine der Variablen konnte ein signifikanter Einfluss nachgewiesen werden. Als zweite Variante des Untermodells Soziodemografie wurde ein Modell berechnet, in dem Einkommen, Ausbildung und Berufsstatus nach dem von Helmert [23] vorgeschlagenen Verfahren zu einem Sozialschicht-Index verknüpft wurden; auch für diese Variable ließ sich ein Einfluss statistisch nicht nachweisen. Etwaige Morbiditätsunterschiede zwischen Wechslern und Nichtwechslern sind demnach nicht dadurch vermittelt, dass Angehörige höherer sozialer Schichten (als typischerweise „bessere Risiken“) vom Recht der freien Kassenwahl vergleichsweise häufiger Gebrauch machen würden.

Das Untermodell Inanspruchnahme umfasste Indikatoren der ambulant-ärztlichen Versorgung und der Krankenhausbehandlung. Während für die ambulante Versorgung keine Unterschiede festgestellt werden konnten, zeigte sich auch bei Kontrolle auf Einflüsse von Alter und Geschlecht, dass der Anteil der Versicherten, die in den der Befragung vorausgegangenen zwölf Monaten stationäre Behandlung im Krankenhaus in Anspruch genommen hatten, unter den Wechslern deutlich geringer war als unter den Nichtwechsler (Odds Ratio =0,713; 95\%-Konfidenzintervall $[0,499-1,018] ; \mathrm{p}=0,0624)$, und dass die Wechsler durchschnittlich weniger Krankenhausaufenthalte als die Nichtwechsler zu verzeichnen hatten (Odds Ratio=0,729; 95\%-Konfidenzintervall $[0,547-0,970] ; \mathrm{p}=0,0380)$.

Im Untermodell Erkrankungsrisiko erwies sich keine der untersuchten Variablen (Bodymass-Index, körperliche Aktivität, Rauchen, Alkoholkonsum) als ein Merkmal, hinsichtlich dessen sich Wechsler von Nichtwechslern alters- und geschlechtsadjustiert voneinander unterschieden. Wenn demnach Kassenwechsler bessere Versicherungsrisiken waren, so offenbar nicht deshalb, weil sie sich durch eine vergleichsweise gesündere Lebensweise auszeichneten. Die Ergebnisse unserer Untersuchung bieten somit auch keine Grundlage für die Annahme einer im Gesundheitsverhalten begründeten längerfristigen Persistenz der beobachteten Risikodifferenziale.

In das Untermodell Morbidität wurden insgesamt 22 Erkrankungen aufgenommen, die entweder als chronische Krankheiten einzustufen sind oder die zwar akute Ereignisse darstellen, aber üblicherweise mit einer anhaltenden Erhöhung des medizinischen Versorgungsbedarfs verbunden sind, wie z.B. Herzinfarkt oder Schlaganfall. Wenngleich die Auftretenshäufigkeit der einbezogenen Erkrankungen auch nach Alters- und Geschlechtsadjustierung in ihrer überwiegenden Mehrheit bei 
Wechslern geringer war als bei Nichtwechslern, stellte sich - angesichts teilweise sehr kleiner Fallzahlen nicht unerwartet - ein statistisch auffälliges Ergebnis nur für chronische Bronchitis ein. Das Untermodell Morbidität wurde daher in einer zweiten Variante untersucht, in der alle 22 Erkrankungen zu einer dichotomen Variable chronische Morbidität zusammengefasst wurden. In dieser Modellvariante zeigte sich, dass die Wechsler in diesem Sinne auch nach Kontrolle auf Alter und Geschlecht deutlich weniger chronisch krank waren als die Nichtwechsler (Odds Ratio =0,769; 95\%-Konfidenzintervall [0,607-0,974]; $\mathrm{p}=0,0296$ ).

In das Untermodell Kassenmerkmale gelangten die (im Risikostrukturausgleich berücksichtigte) Anzahl der über die Probanden beitragsfrei mitversicherten Familienangehörigen, die Art der Krankenversicherung (wie AOK, EK, BKK, IKK, LKK), der Versichertenstatus (Pflichtmitglied, freiwilliges Mitglied, Rentner, beitragsfrei mitversichertes Familienmitglied) sowie als Beitragssatzindikatoren der Beitragssatz zu Beginn der Beobachtungsperiode (1.1.1996) und die größte jährliche Beitragssatzdifferenz während der Beobachtungsperiode (Zeitraum zwischen dem 1.1.1996 und dem Tag des Interviews). Nur die Beitragssatzindikatoren hatten in diesem Untermodell Bestand. Einen signifikanten Einfluss nach Kontrolle auf Geschlecht und Alter hatten der Beitragssatz zu Beginn der Beobachtungsperiode (Odds Ratio=2,454; 95\%-Konfidenzintervall $[1,842-3,271] ; \quad p<0,0001)$ sowie die größte Beitragssatzdifferenz während der Beobachtungsperiode (Odds Ratio = 3,279; 95\%-Konfidenzintervall [2,325-4,624]; $\mathrm{p}<0,0001$ ).

In das Endmodell wurden somit neben der Variable Alter, die sich in sämtlichen Untermodellen als hochsignifikante Einflussgröße erwiesen hatte, die Variablen chronische Morbidität, Häufigkeit der stationären Krankenhausbehandlung in den letzten zwölf Monaten, Beitragssatz zu Beginn der Beobachtungsperiode sowie die maximale jährliche Beitragssatzdifferenz während der Beobachtungsperiode aufgenommen. Die Variable Geschlecht wurde aus konventionalistischen Gründen mitgeführt. Die Schätzergebnisse für dieses Modell sind Tab. 4 zu entnehmen.

Die Schätzergebnisse des Endmodells bestätigen die Befunde der Untermodelle in allen Aspekten. Auch bei Kontrolle auf alle in den Untermodellen als signifikant identifizierten Einflussfaktoren bleibt die dominierende Rolle des Einflussfaktors Alter erhalten; mit jedem zusätzlichen Lebensjahr nimmt demnach die Wechselwahrscheinlichkeit prozentual um etwa 6\% ab. Der Ein- fluss des Beitragssatzes schlägt für jeden zusätzlichen Prozentpunkt mit einer Erhöhung der Wechselwahrscheinlichkeit um das 2,5-fache zu Buche. Auffällig ist, dass dieser Einfluss in den deskriptiven Befunden nicht zum Tragen kommt und erst in der mehrfaktoriellen Analyse sichtbar wird; dies mag vor allem darin begründet sein, dass die Krankenkassen mit zu Beginn der Beobachtungsperiode sehr hohen Beitragssätzen gleichzeitig Kassen mit überdurchschnittlich hohem Anteil älterer Versicherter waren. Der Einfluss von Beitragssatzveränderungen schlägt sich für jeden zusätzlichen Prozentpunkt sogar in einer Erhöhung der Wechselwahrscheinlichkeit um das 3,5-fache nieder. Dieses Resultat ist freilich vor dem Hintergrund zu sehen, dass die maximalen jährlichen Beitragssatzsprünge im Beobachtungszeitraum bei einer Streubreite zwischen $-0,3$ und $+1,9$ Prozentpunkten im Mittel nur 0,68 Prozentpunkte und nur für $12 \%$ der Studienteilnehmer mehr als 1 Prozentpunkt betrugen. Unter diesen Probanden waren dann allerdings zwei Drittel - alles Versicherte derselben Krankenkasse - von dem maximalen Beitragssatzsprung in Höhe von 1,9\% betroffen. Im Gefolge dieser Beitragserhöhung haben über 20\% der ca. 250 Anfang 1996 bei der betreffenden Krankenkasse versicherten Studienteilnehmer ihre Kasse gewechselt, was die massiven Auswirkungen einer Beitragssatzveränderung in dieser Größenordnung plastisch veranschaulicht. Die beiden die chronische Morbidität und die Inanspruchnahme stationärer Versorgung abbildenden Variablen zeigen gleichfalls einen statistisch signifikanten und deutlich ausgeprägten Einfluss auf die Wechselwahrscheinlichkeit, auch wenn sie in der Stärke ihres Einflusses hinter die Alters- und Beitragssatzvariablen zurücktreten. Das Auftreten (mindestens) einer der als chronisch eingestuften Erkrankungen ist mit einer Reduktion der Wechselwahrscheinlichkeit um ein knappes Viertel verbunden; insoweit bringen Wechsler ein alters- und geschlechtsadjustiert deutlich unterdurchschnittliches Maß an Krankheitslast in ihre neue Krankenkasse ein, deren Ausgabenwirksamkeit zwar hohe Plausibilität für sich beanspruchen darf, durch diesen Befund freilich noch nicht statistisch belegt ist. Bezüglich etwaiger Defizite des RSA aussagekräftiger ist daher das Resultat für den Einfluss der Krankenhaushäufigkeit. Hier ergibt sich, dass jeder zusätzliche Krankenhausaufenthalt in den zurückliegenden zwölf Monaten die Wechselwahrscheinlichkeit um fast $30 \%$ reduziert, womit für die stationär behandelte Morbidität ein risikoentmischender Effekt des Kassenwechsels statistisch bestätigt ist.

Tab. 4 Ergebnisse des Endmodells für den Kassenwechsel KORA-Survey S4 (1999/2001)

\begin{tabular}{|c|c|c|c|c|c|c|c|}
\hline Statistik & Absolut-Term & Alter & Geschlecht ${ }^{1}$ & $\begin{array}{l}\text { chronische } \\
\text { Morbidität }^{2}\end{array}$ & $\begin{array}{l}\text { Anzahl } \\
\text { Krankenhausfälle }\end{array}$ & $\begin{array}{l}\text { max. jährl. } \\
\text { Beitragssatzdifferenz }\end{array}$ & $\begin{array}{l}\text { Beitragssatz } \\
\text { 1.1.1996 }\end{array}$ \\
\hline Parameter-Schätzwert & $-1,6461$ & $-0,0640$ & $-0,0417$ & $-0,2654$ & $-0,3114$ & 1,2418 & 0,9257 \\
\hline Standardfehler & 2,0041 & 0,0048 & 0,1143 & 0,1236 & 0,1455 & 0,1753 & 0,1474 \\
\hline Chi-Quadrat-Statistik & 33,7709 & 177,4013 & 0,1329 & 4,6141 & 4,5832 & 50,1801 & 39,4431 \\
\hline p-Wert & $<, 0001$ & $<, 0001$ & 0,7155 & 0,0317 & 0,0323 & $<, 0001$ & $<, 0001$ \\
\hline Odds Ratio & - & 0,938 & 0,959 & 0,767 & 0,732 & 3,462 & 2,524 \\
\hline 95\%-Konfidenzintervall & - & $0,929-0,947$ & $0,767-1,200$ & $0,602-0,977$ & $0,551-0,974$ & $2,455-4,881$ & $1,890-3,369$ \\
\hline
\end{tabular}

1 Referenzkategorie: Frauen

2 Referenzkategorie: keine chronische Morbidität 


\section{Diskussion und Ausblick}

Der Gesetzgeber hat die auf die Herstellung einer solidarischen Wettbewerbsordnung zielende Freigabe der Kassenwahl mit der Einführung eines Risikostrukturausgleichs flankiert, um den Wettbewerb zwischen den Kassen unter Beibehaltung des Prinzips der solidarischen Finanzierung auf die zentralen gesundheitspolitischen Ziele einer bedarfsgerechten und effizienten $\mathrm{Ge}$ sundheitsversorgung auszurichten. Darüber hinaus sollten mit dem RSA allen Krankenkassen trotz der historisch gewachsenen großen Unterschiede in den Risikostrukturen gleiche Startchancen im Wettbewerb gesichert werden.

Unsere Untersuchung hat gezeigt, dass vor allem jüngere Versicherte ihre Kasse wechseln und dass die Richtung der Kassenwechselbewegungen per saldo klar durch die Beitragssatzunterschiede zwischen den Krankenkassen geprägt ist: „Gewinnerkassen“ sind Kassen mit niedrigen Beitragssätzen, „Verliererkassen“ Kassen mit hohen Beitragssätzen. Dies ist insofern keine Überraschung, als den Krankenkassen aufgrund des gesetzlich vorgeschriebenen Leistungskatalogs nur geringe Spielräume für eine leistungsseitige Profilierung offen stehen, das dominierende Motiv für einen Kassenwechsel im Regelfall also die Absicht sein wird, Beitragsvorteile zu realisieren. Jüngere Versicherte erwirtschaften für ihre Krankenkasse aufgrund ihrer im Vergleich zu den älteren Versicherten im Durchschnitt geringeren Leistungsinanspruchnahme und höheren Beitragszahlungen hohe positive Deckungsbeiträge. Ohne den RSA wären Krankenkassen mit hohen Versichertenverlusten mithin finanziell massiv sanktioniert worden, völlig unabhängig davon, ob ihre hohen Beitragssätze Ausdruck von Unwirtschaftlichkeit oder Folge ihrer dem alten Zuweisungsrecht geschuldeten ungünstigen Risikostruktur sind. Insofern hat die Realität der Kassenwanderung die Notwendigkeit eines RSA nachträglich noch einmal bestätigt.

Darüber hinaus hat unsere Untersuchung aber auch gezeigt, dass Kassenwechsler im Vergleich zu Nichtwechslern auch nach Alters- und Geschlechtsstandardisierung „bessere Risiken“ im Sinne einer niedrigeren Belastung durch chronische Krankheiten und einer geringeren Inanspruchnahme stationärer medizinischer Versorgung und insoweit auch der zu erwartenden durchschnittlichen Leistungsausgaben sind. Unsere Ergebnisse bestätigen insofern die Resultate der Studie von Lauterbach und Wille [20], gehen über diese aber auch ein Stück hinaus: Während Lauterbach und Wille die Inanspruchnahme der Wechsler in einem 12-Monatszeitraum vor dem Kassenwechsel erfasst haben, wurde in unserer Studie die stationäre Inanspruchnahme nach erfolgtem Kassenwechsel für einen retrospektiven Zeitraum von einem Jahr erhoben. Nur bei 37\% aller Wechselfälle lag der Wechseltermin noch innerhalb des Zeitraums, für den die Inanspruchnahme erhoben wurde, bei $63 \%$ aller Fälle lag der Wechseltermin mindestens ein Jahr, bei 35\% mindestens zwei Jahre, bei $17 \%$ mindestens drei Jahre und bei $5 \%$ vier Jahre oder länger zurück. Man wird daher kaum davon ausgehen können, dass sich die Risikoprofile der Wechsler nach ihrem Kassenwechsel wieder rapide an die der Nichtwechsler angleichen, sondern eher eine gewisse Persistenz unterstellen müssen, wie sie etwa in einer Untersuchung in der Schweiz beobachtet worden ist [24]. Nicht in Übereinstimmung stehen unsere Studienergeb- nisse hingegen mit den Resultaten der auf Daten des SOEP gestützten Untersuchungen von Andersen und Schwarze [22], die für die Wechsler in den alten Bundesländern aus deren Angaben über den Umfang ihrer Inanspruchnahme stationärer Versorgung lediglich für 1999 einen unterdurchschnittlichen Beitragsbedarf, für die Summe der fünf Jahre von 1997 bis 2001 hingegen einen deutlich überdurchschnittlichen und zudem für jede Jahrgangskohorte im Zeitablauf tendenziell zunehmenden Beitragsbedarf für die Wechsler ableiten, ohne dass wir für diese Diskrepanz eine Erklärung anbieten können.

Nach unseren Ergebnissen erfolgt somit eine Anreicherung der Versichertenbestände von Kassen mit niedrigen Beitragssätzen um „gute Risiken“ und komplementär dazu eine zunehmende Konzentration der „schlechten Risiken“ bei den Kassen mit hohen Beitragssätzen über jene Risiken hinaus, die im derzeit angewendeten Risikoausgleichsverfahren kompensiert werden. Damit besteht die Gefahr, dass sich gleichsam unter der Oberfläche eines dem Prinzip risikounabhängiger Versicherungsbeiträge verpflichteten Finanzierungssystems als Resultat der Freigabe der Kassenwahl Beitragssatzdifferenziale herausbilden, die stark von den durch den geltenden RSA nicht kompensierten Morbiditätsrisiken beeinflusst werden. Unter diesen Bedingungen ist der Kassenwechsel somit der Herstellung von Beitragsgerechtigkeit im Sinne risikounabhängiger Beiträge abträglich, und er trägt dazu bei, dass die nach dem RSA verbleibenden Beitragssatzunterschiede die Leistungs- und Wirtschaftlichkeitsunterschiede zwischen den Krankenkassen nicht korrekt reflektieren und in der Folge die allokationsverbessernde Funktion des Kassenwettbewerbs geschwächt wird. Die Politik hat Empfehlungen aus der Wissenschaft, den RSA in Richtung einer genaueren Erfassung unterschiedlicher Morbiditätsstrukturen der Kassen zu entwickeln, aufgenommen; nach dem Willen des Gesetzgebers soll der RSA vom 1. Januar 2007 an auf der Grundlage von Klassifikationsmerkmalen durchgeführt werden, die die Morbidität der Versicherten auf der Grundlage von Diagnosen, Indikationen und medizinischen Leistungen unmittelbar berücksichtigen. Damit ist die Politik auch nach den Ergebnissen unserer Untersuchung grundsätzlich auf dem richtigen Weg.

\section{Danksagung}

Die Untersuchungen wurden gefördert durch die GSF.

Der Artikel nimmt besonderen Bezug auf folgende Beiträge dieser Sonderausgabe von Das „Gesundheitswesen“: [25-31].

\section{Literatur}

\footnotetext{
${ }^{1}$ Jacobs K, Reschke P, Cassel D et al. Zur Wirkung des Risikostrukturausgleichs in der gesetzlichen Krankenversicherung. Baden-Baden: Nomos Verlagsgesellschaft, 2002

${ }^{2}$ Buchner F, Wasem J. Needs for further improvement: risk adjustment in the German health insurance system. Health Policy 2003; 65: $21-35$

${ }^{3}$ Van de Ven WPM, Beck K, Buchner F et al. Risk adjustment and risk selection on the sickness fund insurance market in five European countries. Health Policy 2003; 65: 75 - 98

${ }^{4}$ Dunn DL. Applications of health risk adjustment: what can be learned from experience to date? Inquiry 1998; 35: $132-147$
} 
${ }^{5}$ Van de Ven WPM, Ellis RP. Risk adjustment in competitive health plan markets. In: Culyer AL, Newhouse JP (eds) (Hrsg). Handbook of health economics, Vol. 1. Amsterdam: Elsevier, 2000: 735-845

${ }^{6}$ Felder S. Wehret den Anfängen! Gegen Morbiditätsfaktoren im Risikostrukturausgleich. Wirtschaftsdienst 2001; 4: 198-201

${ }^{7}$ Breyer F, Heieck M, Lorenz M. Determinants of health care utilization by German sickness fund members: with application to risk adjustment. Health Econ 2003; 12: 367-376

${ }^{8}$ Müller J, Schneider W. Mitgliederbewegungen und Beitragssätze in Zeiten des Kassenwettbewerbs. Ein erster empirischer Befund nach Inkrafttreten der Wahlfreiheit. Arbeit und Sozialpolitik 1997; 3-4: $11-24$

${ }^{9}$ Müller J, Schneider W. Entwicklung der Mitgliederzahlen, Beitragssätze, Versichertenstrukturen und RSA-Transfers in Zeiten des Kassenwettbewerbs. Empirische Befunde im zweiten Jahr der Kassenwahlfreiheit. Arbeit und Sozialpolitik 1998; 3-4: 10-32

${ }^{10}$ Müller J, Schneider W. Entwicklung der Mitgliederzahlen, Beitragssätze, Versichertenstrukturen und RSA-Transfers in Zeiten des Kassenwettbewerbs. Empirische Befunde im zweiten Jahr der Kassenwahlrechte. Arbeit und Sozialpolitik 1999; 3-4: 20-39

${ }^{11}$ Greß S, Braun B, Groenewegen P et al. Erfahrungen mit der freien Krankenkassenwahl in Deutschland und in den Niederlanden. Sozialer Fortschritt 2002; 4: 97-101

${ }^{12}$ Greß S, Groenewegen P, Kerssens J et al. Free choice of sickness funds in regulated competition: evidence from Germany and the Netherlands. Health Policy 2002; 60: 235-254

${ }^{13}$ Böcking W, Göpfert P, Merker N et al. The relevance of price in a customer's choice of statutory health insurance providers in Germany. Zeitschrift für Gesundheitswissenschaften 2002; 10: 242 - 251

${ }^{14}$ Pittrow D, Böcking W, Kirch W. Determinanten des Kassenwechsels in der gesetzlichen Krankenversicherung. Welche Bedeutung kommt den Strukturfaktoren der Versicherungen zu? Gesundheitsökonomie und Qualitätsmanagement 2003; 8: 24-30

${ }^{15}$ Schut FT, Greß S, Wasem J. Consumer price sensitivity and social health insurer choice in Germany and the Netherlands. Int J Health Care Finance and Econ 2003; 3: 117-138

${ }^{16}$ Andersen HH, Schwarze J. GKV '97. Kommt Bewegung in die Landschaft? Eine empirische Analyse der Kassenwahlentscheidungen. Arbeit und Sozialpolitik 1998; 9-10: 11-23

${ }^{17}$ Andersen HH, Schwarze J. Kassenwahlentscheidungen in der GKV. Eine empirische Analyse. Arbeit und Sozialpolitik 1999; 5-6: 10-23

18 Schwarze J, Andersen HH. Kassenwechsel in der gesetzlichen Krankenversicherung. Welche Rolle spielt der Beitragssatz? Schmollers Jahrbuch 2001; 121: $581-602$

${ }^{9}$ Knaus $\mathrm{T}$, Nuscheler $\mathrm{R}$. Incomplete risk adjustment and adverse selection in the German public health insurance system. WZB Discussion Papers FS IV 02 - 27. Berlin: WZB, 2002

${ }^{20}$ Lauterbach KW, Wille E. Modell eines fairen Wettbewerbs durch den Risikostrukturausgleich. Sofortprogramm „Wechslerkomponente und solidarische Rückversicherung“ unter Berücksichtigung der Morbidität. Gutachten im Auftrag des Verbandes der Angestellten-Krankenkassen e.V. (VdAK), des Arbeiter-Ersatzkassen-Verbandes e.V., des AOK-Bundesverbandes (AOK-BV) und des IKK-Bundesverbandes (IKKBV). Köln/Mannheim, 2001 (unveröffentlicht)

${ }^{21}$ Winkelhake O, Miegel U, Thormeier K. Die personelle Verteilung von Leistungsausgaben in der gesetzlichen Krankenversicherung 1998 und 1999. Sozialer Fortschritt 2002; 3: 58-61

22 Andersen HH, Grabka MM, Schwarze et al. Wechslerprofile - Risikoprofile. Relativer Beitragsbedarf der Kassenwechsler 1997-2001. Arbeit und Sozialpolitik 2002; 7-8: 19-32

${ }^{23}$ Helmert U. Soziale Ungleichheit und Krankheitsrisiken. Augsburg: Maro Verlag, 2003

${ }^{24}$ Beck K, Keller B. Risikoselektion in Hausarztmodellen. Ein Plädoyer für differenzierte Risikokorrektur. Manag Care 1998; 4: 30 - 33

${ }^{25}$ Löwel H, Döring A, Schneider A et al. The MONICA Augsburg surveys basis for prospective cohort studies. Gesundheitswesen 2005; 67 S1: S13-S18

${ }^{26}$ Wichmann HE, Gieger C, Illig T. KORA-gen - Resource for population genetics, controls and a broad spectrum of disease phenotypes. Gesundheitswesen 2005; 67 S1: S26-S30

${ }^{27}$ Löwel H, Meisinger C, Heier M et al. The population-based acute myocardial infarction (AMI) registry of the MONICA/KORA study region of Augsburg. Gesundheitswesen 2005; 67 S1: S31 - S37

${ }^{28}$ Mielck A, Reisig V, Rathmann W. Health inequalities among persons with type 2 diabetes: the example of intermittent claudication. Gesundheitliche Ungleichheit bei Typ 2 Diabetes. Gesundheitswesen 2005; 67 S1: S137-S143

${ }^{29}$ Eller M, Satzinger W, Holle R et al. Disease-Management-Programme in Deutschland. Erste Reaktionen der Diabetiker. Gesundheitswesen 2005; 67 S1: S144-S149

${ }^{30}$ Lengerke Tv, Happich M, Reitmeir P et al. Utilization of out- and inpatient health services by obese adults: a population - based study in the Augsburg region, Germany. Gesundheitswesen 2005; 67 S1: S150-S157

${ }^{31}$ Icks A, Rathmann W, Haastert B et al. Cost-effectiveness of type 2 diabetes screening: results from recently published studies. Gesundheitswesen 2005; 67 S1: S167-S171 